Der folgende Artikel setzt sich pointiert mit einem Thema auseinander, das die Gemüter breiter Bevölkerungskreise bewegt und in den Medien immer wieder hohe Wellen wirft. Für die Ärzteschaft sind Zwangsausschaffungen insofern von besonderer Bedeutung, als medizinische und ethische Aspekte und Erwägungen dabei eine zentrale Rolle spielen. Kollege Michel Romanens bezieht denn aus ärztlicher Sicht auch unmissverständlich Stellung, was im Ressort «Tribüne» der SÄZ durchaus möglich sein soll. Da er sich kritisch zur Arbeit des Instituts für Rechtsmedizin der Universität Zürich (IRMZ) und weiterer involvierter Organe äussert, haben wir dem IRMZ und der Staatsanwaltschaft Gelegenheit zur Stellungnahme gegeben. Beide Stellen haben unter Hinweis auf das noch laufende Verfahren im Fall des im März 2010 bei einem Ausschaffungsversuch verstorbenen K. A. auf einen Kommentar zum Beitrag verzichtet. Die Redaktion

\title{
Tod durch Ausschaffung: Bemerkung zum Level IV
}

Viktor Györffy

Der Autor ist Rechtsanwalt und vertritt die Familie von K. A., der im März 2010 bei einem Ausschaffungsversuch verstorben ist.
Korrespondenz:

Viktor Györffy

Rechtsanwalt

Gartenhofstrasse 15

Postfach 9819

CH-8036 Zürich

gyoerffy@advogar.ch

\section{Begleitung einer Ausschaffung durch Ärzte: juristische Aspekte}

Welches juristische Risiko trägt ein Arzt, der eine Zwangsausschaffung begleitet? Wenn ein Häftling im Rahmen einer Zwangsausschaffung einen gesundheitlichen Schaden erleidet oder gar stirbt, dann wird die Justiz zu klären haben, ob sich der Arzt strafbar gemacht hat und ob er für den entstandenen Schaden haftet. Strafrechtlich wird nach einem solchen Vorfall zu prüfen sein, ob sich Personen, die in die Ausschaffung involviert gewesen sind, der fahrlässigen Körperverletzung oder Tötung schuldig gemacht haben. Haftungsrechtlich stellt sich die Frage, ob eine involvierte Person, Behörde oder Organisation für den Schaden, der dem Häftling oder seinen Hinterbliebenen entstanden ist, Ersatz leisten muss.

Der Massstab für die Verantwortlichkeit hängt nicht zuletzt von den fachlichen Kenntnissen und Pflichten der involvierten Personen ab. Ein Arzt ist dabei naturgemäss besonders exponiert. Von ihm wird am ehesten verlangt, dass er in der Lage ist, die mit einer Zwangsausschaffung verbundenen Risiken einzuschätzen. Er kann den Gesundheitszustand des Betroffenen eruieren und eingreifen, wenn sich eine für die Gesundheit gefährliche Situation einstellt. Zudem spielen bei den Pflichten eines Arztes immer auch die ethischen Richtlinien für die ärztliche Tätigkeit eine Rolle. Diesen kann sich der Arzt unabhängig vom konkreten Auftrag nicht entziehen. Die grundlegenden Pflichten können über den konkreten Auftrag hinausgehen oder mit jenem kollidieren. Wie weit seine Verantwortung für den Gesundheitszustand des Auszuschaffenden im jeweiligen Fall geht, ist unter diesen Umständen schwer festzumachen. Klar ist nur, dass juristische Risiken lauern. Es wird dem Arzt nur begrenzt möglich sein, den Gesundheitszustand des Auszuschaffenden zu beurteilen. Inwieweit kann er sich auf die ihm vorgelegten Informationen verlassen, inwieweit muss er selbst Abklärungen treffen? Wie kann er das Vertrauen des Betroffenen erlangen? Mit welchen Problemen muss er bei einer Ausschaffung rechnen und über welche besonderen Fachkenntnisse muss er verfügen, um diesen begegnen zu können? Wann muss er während der Ausschaffung zum Schutz des Auszuschaffenden eingreifen?

Zur Illustration sei dazu ein konkreter Fall erwähnt: Am 3. März 1999 erstickte Khaled Abuzarifa, nachdem er zwecks Ausschaffung auf einen Rollstuhl gefesselt und sein Mund mit Klebeband zugeklebt wurde. Es wurden Strafverfahren wegen fahrlässiger Tötung gegen drei Polizisten und einen anwesenden Arzt eröffnet. Zwei Polizisten wurden erstinstanzlich freigesprochen, der Gruppenchef verstarb noch während der Ermittlungen. Rechtskräftig verurteilt wurde einzig der Arzt, zu 5 Monaten Gefängnis bedingt. Er hatte der Fesselung und Knebelung beigewohnt und diese als unbedenklich eingestuft. Das Urteil wurde letztinstanzlich vom Bundesgericht bestätigt.

\section{Begleitung einer Ausschaffung durch Ärzte: medizinische Aspekte}

Michel Romanens

\section{Hintergrund}

Zwangsausschaffungen gelten als letztes Mittel im Ausweisungsvollzug. Dieser kennt verschiedene Grade der Zwangsmassnahmen. Mit Level IV wird die auszuschaffende Person praktisch vollständig handlungsunfähig und ist während dieser Zeit der Staatsgewalt wehrlos ausgeliefert.

\section{Fragestellung}

Am 17. März 2010 starb Herr K. A., geb. 1980, im Flughafen Zürich, als er ausgeschafft werden sollte. Er befand sich seit mindestens 40 Tagen im Hungerstreik und war gemäss Bodymass-Index im Grenzbereich zur Kachexie angelangt. Haben die ausschaffenden Institutionen den Vollzug adäquat ausgeführt? 


\section{Bemerkungen zur Diagnose}

In den Medien wurde die Behauptung aufgestellt, dass Herr K. A. wegen einer schweren Herzkrankheit gestorben sei, also mehr oder weniger zufällig ohne Ursache im Ausschaffungsvollzug selbst. Dies ist eine Schutzbehauptung. Gemäss Bericht des Instituts für Rechtsmedizin der Universität Zürich soll Herr K. A. an einer tubulären Kardiomyopathie gelitten haben, da fibrotische subendokardiale Veränderungen sowie eine Fibrose des Septalastes vorlagen. Aus diesen Veränderungen, die keineswegs spezifisch sind, wurde die Diagnose einer schweren hypertrophen Kardiomyopathie (HCM) konstruiert.

Ähnlich argumentierte ein Pathologe der Universität Bern, der für diese Frage beigezogen wurde, wonach der «Dysarray pathognomonisch für die HCM» sei. Dies ist nachweislich wissenschaftlich nicht haltbar, denn ein Dysarray kann bei einer Vielzahl anderer Herzkrankheiten auftreten [1]. Zwar können spontan tödliche Arrhythmien bei der HCM auftreten, dazu benötigt es jedoch in den meisten Fällen ein Substrat (= erhebliche pathologische Veränderungen als Grundlage für letale Arrhythmien). Aus dem Pathologiebericht geht jedoch keineswegs hervor, dass eine schwere HCM vorlag, das Herzgewicht war zudem normal (270 g), und die Vorhofgrösse (als Ausdruck der diastolischen Dysfunktion bei schwerer HCM) wurde erst gar nicht beschrieben. Zwar kann der Herzmuskel durch Fasten an Masse abnehmen, entsprechend müssten dann die fibrotischen Veränderungen relativ zunehmen und sich nicht nur auf septal subendokardiale Bezirke beschränken.

Dass die HCM nur mit kardialer Magnetresonanz erkannt werden kann, ist eine weitere Schutzbehauptung aus dem Gutachten der Zürcher Rechtsmediziner. Die HCM ist mit einer Sensitivität und Spezifität von $90 \%$ im Ruhe-Elektrokardiogramm zu erkennen [2]. Zudem ist die Familienanamnese für plötzlichen Herztod, ein typisches Feature der HCM, bei Herrn K. A. unauffällig, und weiterführende Abklärungen wie Gentests wurden entgegen der Erwähnung dieser Möglichkeit durch den Berner Pathologen nicht angeordnet. Fazit: Die Diagnose der HCM steht bei diesem Patienten keineswegs fest, es ist sogar denkbar, dass der Hungerstreik selbst teilweise die PathologieBefunde erklärt (siehe auch «Bemerkungen zur Todesursache»).

\section{Bemerkungen zur Risikoaufklärung}

Das Vorgehen des Gefängnisarztes muss auf Basis einer ausreichenden Aufklärung erfolgen (informed consent). Gemäss den ethischen Richtlinien muss die inhaftierte Person durch den Arzt in objektiver Art und Weise und wiederholt über die möglichen Risiken von längerem Fasten aufgeklärt werden. Die volle Urteilsfähigkeit der betreffenden Person muss von einem ausserhalb der Anstalt tätigen Arzt bestätigt worden sein. Das Vorgehen im Fall K. A. genügt den Richtlinien nicht. Sicher ist, dass eine Abklärung der
Urteilsfähigkeit durch einen externen Arzt unterblieben ist. Dass die Aufklärung durch den Gefängnisarzt genügend gewesen ist, erscheint zweifelhaft. Jedenfalls ist eine genügende Aufklärung nicht belegt, insbesondere nicht eine Aufklärung mit Blick auf die durch den Hungerstreik verursachten besonderen Risiken einer Ausschaffung Level IV. Die Zürcher Rechtsmediziner schreiben, der Gefängnisarzt habe K. A. vermutlich auf die Gefahren seines Verhaltens aufmerksam gemacht. Sie haben sich also nicht die Mühe gemacht, vertieft abzuklären, ob und wie aufgeklärt wurde. Unverständlicherweise sehen sie sich aber dennoch zu dem Schluss in der Lage, der Gefängnisarzt habe sich bezüglich der Respektierung des Entscheides des Betroffenen zum Hungerstreik, der notwendigen Aufklärung über die Gefahr desselben sowie der Absicherung, dass ihm regelmässig zu essen und zu trinken angeboten wurde, entsprechend den Richtlinien verhalten.

\section{Bemerkungen zur Notfallsituation}

Herr K. A. wurde für die Level-IV-Ausschaffung vollständig an einen Transportstuhl gefesselt (siehe nachgestellte Situation auf dem Bild bzw. Video [3]. Der Spuckschutz über dem Kopf sowie die Art der Fesselung mit inkliniertem Oberkörper gestatten es nicht, eine Gefahrensituation für Herrn K. A. rasch zu erkennen. Tritt ein Kreislaufstillstand auf, kann dies völlig unbemerkt stattfinden, da der Tonusverlust bei Kreislaufstillstand wegen der Fesselung nicht zu einer Veränderung der Körperhaltung führt. Ferner kann die Pupillenreaktion durch den Spuckschutz ungenügend beurteilt werden. Die Art der Fesselung gestattet es ferner nicht, die typischen Orte der Pulspalpation (Karotiden, A. radialis) zur Feststellung einer noch vorhandenen Kreislaufaktion zu benutzen. Im Falle der Feststellung einer Notsituation muss Herr K. A. zuerst von den Fesseln befreit werden, bevor er überhaupt adäquat reanimiert werden kann. Dies dürfte mehrere Minuten in Anspruch nehmen. Gemäss dem Bericht der Zürcher Rechtsmediziner lagen zudem keine Verletzungen des Brustraumes vor, die eine effiziente Herzmassage belegen würden. Herr K. A. starb somit mehr oder weniger unbemerkt und ohne adäquate medizinische Hilfestellung.

\section{Bemerkungen zur Weitergabe von Informationen}

Es ist bislang nicht geklärt, inwieweit den Beamten, die die Ausschaffung ausführten, bekannt war, dass K. A. im Hungerstreik war. Nicht zur Klarheit beigetragen hat auf jeden Fall die Informationspolitik des ärztlichen Dienstes des Flughafengefängnisses. Die ethischen Richtlinien halten diesbezüglich Folgendes fest: Falls der Arzt zu einer inhaftierten Person gerufen wird, der eine Zwangsmassnahme bevorsteht, muss er eine neutrale und professionelle Haltung einnehmen und den Patienten darüber informieren, dass er ihm zur Verfügung steht und dass keine medizinische 


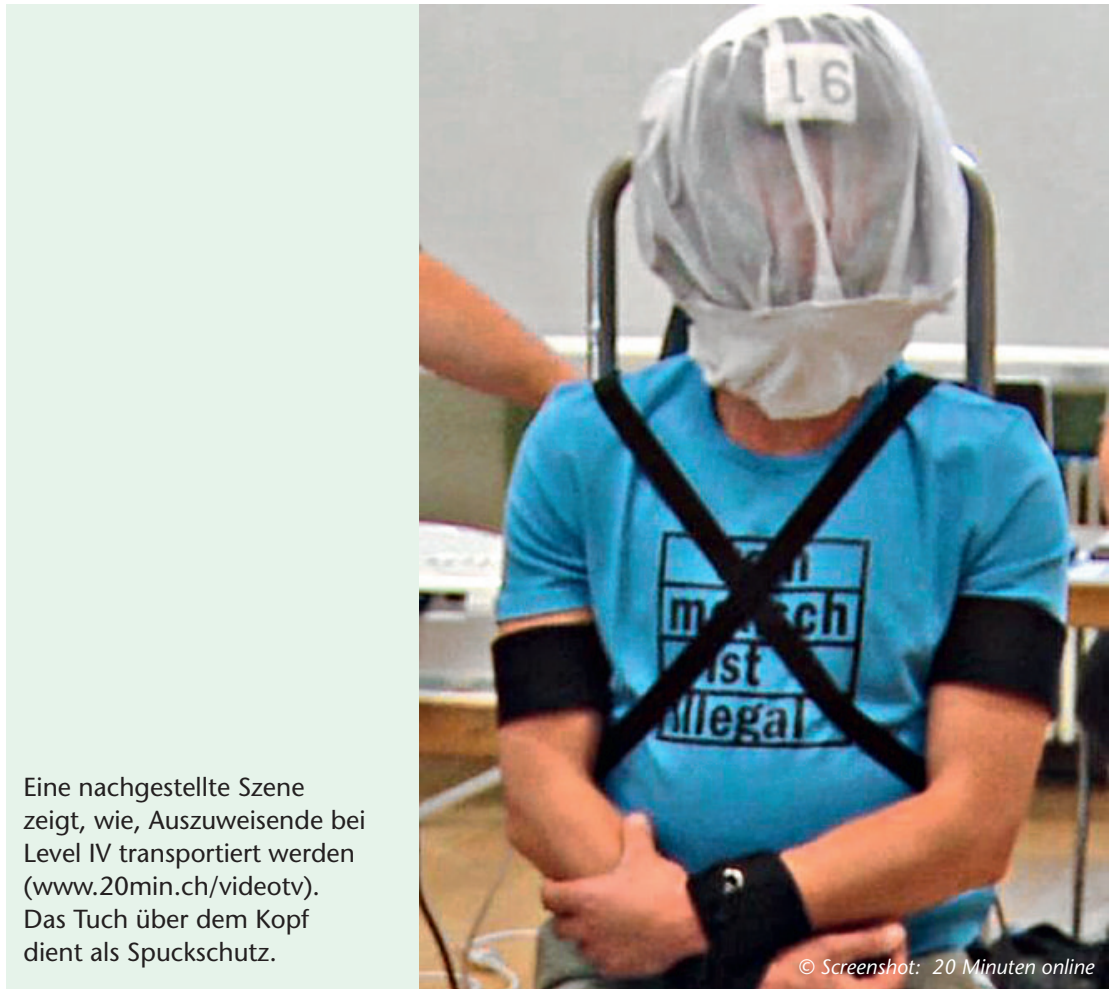

Handlung ohne sein Einverständnis durchgeführt wird. Gelangt der Arzt zur Überzeugung, dass die zur Ausführung der Massnahme eingesetzten Mittel (Knebelung, enge und langfristige Fesselung usw.) für den Patienten eine unmittelbare und erhebliche gesundheitliche Gefahr darstellen, muss er unverzüglich die zuständigen Behörden darüber informieren, dass er, falls auf die vorgesehenen Mittel nicht verzichtet wird, keine medizinische Verantwortung übernimmt und dass er jede weitere Mitwirkung verweigert. Eine andere Möglichkeit besteht darin, dass der Gefängnisarzt sich bei der Behörde um eine Entbindung von der ärztlichen Schweigepflicht bemüht, um detailliert Auskunft geben zu können. Im konkreten Fall überliess es der Gefängnisarzt der Pflegedienstleiterin, ein Faxformular auszufüllen, auf dem die Behörden nach dem Gesundheitszustand der Auszuschaffenden gefragt hatten. Sie trug darauf zu allen Personen «i.O.» und « $\varnothing$ bekannt» ein, ausser bei einem Insassen, bei dem sie die Medikation, die er aufgrund von Nierenproblemen benötigte, aufführte.

\section{Bemerkungen zur Todesursache}

Der mehrwöchige Hungerstreik von Herrn K. A. ist schon per se eine Kontraindikation für die Durchführung von Zwangsmassnahmen auf dem Level IV [4]. Es ist davon auszugehen, dass die durch die Ausschaffung erzeugte Stress-Situation zu einer erheblichen Katecholamin-Ausschüttung geführt hat, die auch ohne das Vorliegen einer HCM zu letalen Arrhythmien bei unterernährten Personen führen kann.

\section{Schlussfolgerungen und Implikationen}

Ausschaffungen mit Level IV können aus ethischmedizinischen Gründen nicht toleriert werden. Die Überwachung scheitert vorab an der Ungewissheit über den Gesundheitszustand der auszuschaffenden Person. Ein Arzt, der eine Ausschaffung begleitet oder eine Verantwortung bei einer Ausschaffung übernimmt, kann weder sicher sein, dass er über alle bereits erhobenen Informationen zum Gesundheitszustand verfügt (sind doch die behandelnden Ärzte und namentlich auch der Gefängnisarzt an das Arztgeheimnis gebunden), noch wird er die Möglichkeit haben, den Gesundheitszustand des Betroffenen selbst genügend abklären zu können. Er befindet sich zudem in einem nicht auflösbaren Rollenkonflikt, da er gleichzeitig der ausschaffenden Behörde und dem Auszuschaffenden dienen soll. Es wird sich so auch kaum ein Vertrauensverhältnis zum Auszuschaffenden aufbauen lassen. Damit müsste der Arzt bereit sein, Verantwortung für einen Menschen zu übernehmen, dessen Gesundheitszustand er nicht zureichend kennt, und sich in ein Spannungsfeld zu begeben zwischen den Interessen der Behörde, die ausschaffen will, und dem Betroffenen, der hierbleiben und unbeschadet bleiben will.

Eine adäquate medizinische Überwachung, die ein medizinisches Gebot in solchen Situationen darstellt, ist damit unmöglich. Unmöglich ist auch die rechtzeitige Überführung der Person in eine reanimationstaugliche Position. Daraus folgt, dass bei Auftreten eines Kreislaufkollapses die Überlebenschancen gering sind. Für den Todesfall von K. A. hat die ausführende Instanz die volle Verantwortung zu übernehmen. Die ethischen Richtlinien geben klare Leitplanken vor [5], die gerade auch in solchen Ausnahmesituationen, wie sie bei einer Ausschaffung mit Level IV vorliegen, uneingeschränkt zu gelten haben. Eine völlig unabhängige Beurteilung der Ereignisse um den Tod durch Ausschaffung von Herrn K. A. liegt bisher immer noch nicht vor. Die ausschaffende Institution hat im Fall von Herrn K. A. offensichtlich versagt. Die Sicherheit auszuschaffender Personen mit Level IV ist nicht gewährleistet. Die Teilnahme von Ärztinnen und Ärzten an solchen Prozeduren muss abgelehnt werden.

\section{Literatur}

1 Maron B, Anan T, Roberts W. Quantitative analysis of the distribution of cardiac muscle cell disorganization in the left ventricular wall of patients with hypertrophic cardiomyopathy. Circulation. 1981 63:882-94.

2 www.jecgonline.com/article/S0022-0736(10)00379-1/ abstract

3 www.20min.ch/news/schweiz/story/27102369

4 www.healthtree.com/articles/eating-disorders/ anorexia/health-effects/

5 www.fmh.ch/files/pdf4/Anhang1_dt_2010.pdf 